\title{
Carcinoembryonic antigen-related cell adhesion molecule 1 modulates vascular remodeling in vitro and in vivo
}

\author{
Andrea Kristina Horst,, ${ }^{1,2}$ Wulf D. Ito,,$^{3,4}$ Joachim Dabelstein, ${ }^{3,4}$ Udo Schumacher, ${ }^{5}$ Heike Sander, ${ }^{1}$

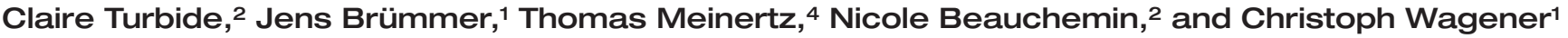 \\ 1Department of Clinical Chemistry, Center of Clinical Pathology, University Medical Center Hamburg-Eppendorf, Hamburg, Germany. \\ 2McGill Cancer Center, McGill University, Montreal, Quebec, Canada. ${ }^{3}$ Department of Medicine II, Angiology and Cardiology, \\ University Medical Center Schleswig-Holstein, Campus Lubeck, Lubeck, Germany. ${ }^{4}$ Center of Cardiology and Cardiovascular Surgery and \\ ${ }^{5}$ Department of Anatomy II, Experimental Morphology, University Medical Center Hamburg-Eppendorf, Hamburg, Germany.
}

\begin{abstract}
Carcinoembryonic antigen-related cell adhesion molecule 1 (CEACAM1), a cellular adhesion molecule of the Ig superfamily, is associated with early stages of angiogenesis. In vitro, CEACAM1 regulates proliferation, migration, and differentiation of murine endothelial cells. To prove that CEACAM1 is functionally involved in the regulation of vascular remodeling in vivo, we analyzed 2 different genetic models: in Ceacam $1^{-/-}$mice, the Ceacam 1 gene was deleted systemically, and in CEACAM $1^{\text {endo+ }}$ mice, CEACAM1 was overexpressed under the control of the endothelial cell-specific promoter of the Tie2 receptor tyrosine kinase. In Matrigel plug assays, Ceacam $1^{-/-}$mice failed to establish new capillaries whereas in CEACAM1 ${ }^{\text {endo+ }}$ mice the implants were vascularized extensively. After induction of hind limb ischemia by femoral artery ligation, Ceacam $1^{-/-}$mice showed significantly reduced growth of arterioles and collateral blood flow compared with their WT littermates. In agreement with a causal role of CEACAM1 in vascular remodeling, CEACAM1 ${ }^{\text {endo+ }}$ mice exhibited an increase in revascularization and collateral blood flow after arterial occlusion. Our findings indicate that CEACAM1 expression is important for the establishment of newly formed vessels in vivo. Hence CEACAM1 could be a future target for therapeutic manipulation of angiogenesis in disease.
\end{abstract}

\section{Introduction}

Blood vessel outgrowth and remodeling requires a well-orchestrated network of vascular growth factors and their cognate receptors. Under both physiological and pathological conditions, vascular remodeling is a key event supporting the development and regeneration of organs or the progression of malignant disease (1-3). In adults, vascular growth mainly occurs through angiogenesis, the sprouting of preexisting vessels, and arteriogenesis, the remodeling of preexisting collateral anastomoses (4). In both processes, newly formed vessels enhance nutrient supply, overcome hypoxia produced by rapid cell turnover, and aid entry of circulating accessory cells or attachment of metastatic cells. Whereas proper angiogenesis and arteriogenesis are required for resolution of certain clinical conditions, such as ischemic heart disease or stroke, such processes are far less desirable in other conditions, such as tumor growth or metastasis, and have become targets for therapeutic intervention $(3,5-7)$.

In addition to growth factors and their receptors, in particular from the VEGF and Tie families, adhesion molecules such as integrins, vascular endothelial cadherin (VE-cadherin), and Ig superfamily (IgSF) members including PECAM1 and endothelial cell-selective adhesion molecule participate in the regulation of vascular remodeling $(5,8-11)$. We and others reported that the IgSF molecule carcinoembryonic antigen-related cell adhesion

Nonstandard abbreviations used: Ang-1, angiopoietin-1; CEACAM1, carcinoembryonic antigen-related cell adhesion molecule 1; CEACAM1-L, CEACAM1 with a long cytoplasmic domain; IgSF, Ig superfamily; lacZ, $\beta$-galactosidase reporter gene.

Conflict of interest: The authors have declared that no conflict of interest exists.

Citation for this article: J. Clin. Invest. 116:1596-1605 (2006). doi:10.1172/JCI24340 molecule 1 (CEACAM1) is associated with early stages of angiogenesis: CEACAM1 is expressed in newly formed blood vessels on the fetal-maternal interface in both rodents and humans; on microvessels of a variety of human tumors and of regenerating tissues, such as endometrium or granuloma tissue after wounding; on endothelial progenitor cells; and in stenotic aortic valve tissue (12-16). Notably, CEACAM1 exhibits a disease-specific expression pattern on tumoral endothelium in vivo, as demonstrated by $\mathrm{Oh}$ et al. (17), and appears to be involved in vascular reprogramming in Kaposi sarcoma (18). Cardiac hypoxia results in upregulation of CEACAM1 expression simultaneously with other angiogenic growth factors (19).

In primary endothelial cell culture, human CEACAM1 enhances endothelial cell sprouting and migration, acting synergistically with VEGF (13). VEGF treatment induces upregulation of CEACAM1 on both protein and mRNA levels, suggesting that CEACAM1 may be a target of VEGF-induced signal transduction (13, 20-22). Also, CEACAM1-induced migration and invasion of human endothelial cells are abolished by anti-CEACAM1-specific antibodies or by silencing CEACAM1 expression via siRNA $(13,16)$.

Despite considerable in vitro evidence for a role of CEACAM1 in the regulation of angiogenesis, little is currently known about angiogenic functions of CEACAM1 in vivo. The present study was designed to elucidate the in vivo function of CEACAM1 in vascular growth. For this purpose, we investigated the function of murine CEACAM1 in angiogenesis and arteriogenesis in different genetically altered mouse strains. In our models we report that (a) expression of intact CEACAM1 in murine endothelial cells was required for the regulation of endothelial proliferation and invasion; (b) additional endothelial expression of CEACAM1 
A

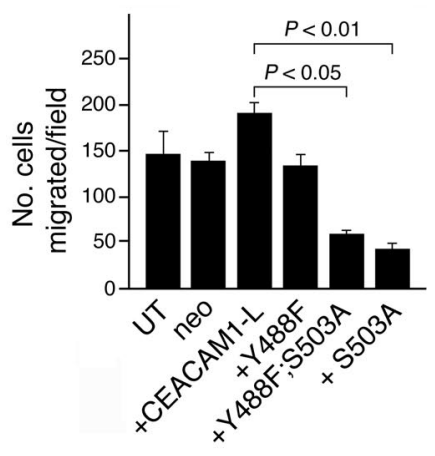

B

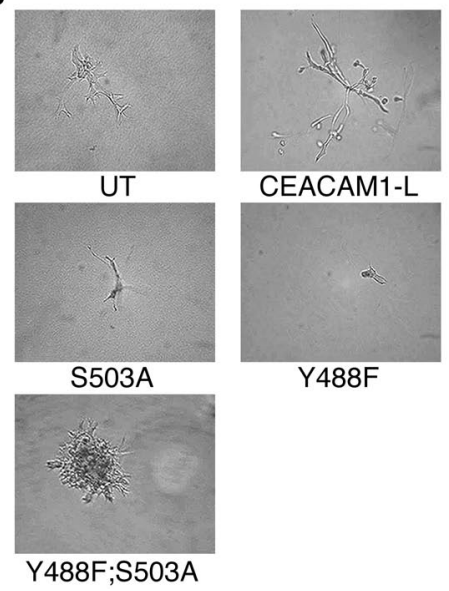

Figure 1

Effects of CEACAM1 expression on the murine endothelial cell line SVEC4-10. (A) Two-dimensional Matrigel transwell assay with different CEACAM1-infected SVEC4-10 clones. After retrovirally mediated infections, 3 clones per CEACAM1 mutant were selected and subjected to a transmigration assay with NIH3T3 fibroblast-conditioned medium as a chemoattractant in the bottom chamber. All assays were carried out in triplicate, and the number of migrated cells was averaged. UT, untransfected SVEC4-10 cells; neo, mock-infected. (B) Three-dimensional fibrin assay with CEACAM1-infected SVEC4-10 cells. SVEC4-10 cells expressing different forms of CEACAM1-L were grown in a 3-dimensional fibrin matrix for 3 days, and endothelial cell protrusions were documented. Experiments were carried out in triplicate with 3 different clones per mutant. Representative images of 1 clone are shown. Magnification, $\times 200$. enhanced vascular remodeling in vivo; (c) specific blocking of CEACAM1 by a monoclonal anti-CEACAM1 antibody led to regression and destabilization of endothelial cell networks; and (d) lack of endothelial CEACAM1 expression in Ceacam $1^{-/-}$mice led to defective vascular remodeling in vivo, whereas endothelial overexpression of CEACAM1 in CEACAM1 $1^{\text {endo+ }}$ mice induced extensive vascular growth. Together, these results suggest that CEACAM1 plays a significant role in angiogenesis and arteriogenesis in vivo.

\section{Results}

CEACAM1 regulates endothelial cell motility and invasion. Based on our in vitro data that human CEACAM1 acts as an angiogenic molecule $(12,13,15,16,20-22)$, we established an in vitro model to show that CEACAM1 affects endothelial cell behavior in the mouse. Our particular interest was set on endothelial cell proliferation and invasion, since CEACAM1 signaling has significant impact on invasive and proliferative properties in different cellular contexts: CEACAM1 regulates tumor cell growth in vitro and in vivo, regulates epithelial lumen formation, colocalizes with integrin $\alpha_{V} \beta_{3}$ at the invasive front of the extravillous trophoblast and malignant melanomas, and forms a coreceptor complex with the insulin and EGF receptors (23-27). Most of these processes are dependent on tyrosine and serine phosphorylation within conserved signaling motifs in the major CEACAM1 isoform, CEACAM1 with a long cytoplasmic domain (CEACAM1-L). To show a functional implication for signal transduction through CEACAM1-L in murine endothelia, we used the murine

\section{Figure 2}

Generation and characterization of CEACAM1 endo+ mice. (A) Transgenic construct for the generation of CEACAM1 $1^{\text {endo+ }}$ transgenic mice. The Ceacam1-L cDNA with an additional SV40 polyadenylation (pA) signal was cloned into the Sse8387 I and Mlu I restriction sites, downstream of the Tie2 promoter and upstream of a Tie2 intronic enhancer. The arrow indicates the position and length of the probe used for genotyping transgenic mice by Southern blots and the fragment that was amplified by PCR. (B) Southern blot of CEACAM1 ${ }^{\text {endo+ }}$ transgenic founder lines. Microinjection of oocytes with the construct depicted in A yielded 5 founder lines, as shown by Southern Blot analysis. Con, control. (C) Confirmation of endothelial CEACAM1 overexpression on primary endothelial cells from lung by flow cytometric analysis using antiPECAM1 and anti-CEACAM1 double labeling in CEACAM1endo+ mice. endothelial cell line SVEC4-10, which expresses CEACAM1-L only in very low amounts. We generated SVEC4-10 transfectants overexpressing either WT CEACAM1-L or different mutants of CEACAM1-L with mutations in one of its cytoplasmic tyrosine $\left(\mathrm{Tyr}_{488}\right)$ or serine $\left(\mathrm{Ser}_{503}\right)$ residues. In 2 - and 3 -dimensional ECM assays, we analyzed the contribution of CEACAM1-L to endothelial cell differentiation, migration, and invasion using Matrigel and fibrin as ECM components. Quantitative analyses of the effects of CEACAM1 overexpression in SVEC4-10 cells are summarized in Figure 1A: CEACAM1 overexpression led to increased migration through a 2-dimensional ECM, whereas conversion of $\mathrm{Tyr}_{488}$ to phenylalanine (Y488F) led to a marked reduction of the motile capacities of transfected SVEC4-10 cells. In addition, conversion of $\operatorname{Ser}_{503}$ to alanine (S503A) abrogated cellular invasion, similar to the effects observed after replacement of both $\mathrm{Tyr}_{488}$ by phenylalanine and $\operatorname{Ser}_{503}$ by alanine. In a 3-dimensional fibrin culture, CEACAM1-L-infected SVEC4-10 cells showed enhanced invasion and differentiation into tubelike structures compared with untransfected cells (Figure 1B). Similar to the data obtained in the 2-dimensional Matrigel transmigration assay, SVEC4-10 cells expressing mutant forms of CEACAM1-L with mutations in their cytoplasmic $\mathrm{Tyr}_{488}$ or

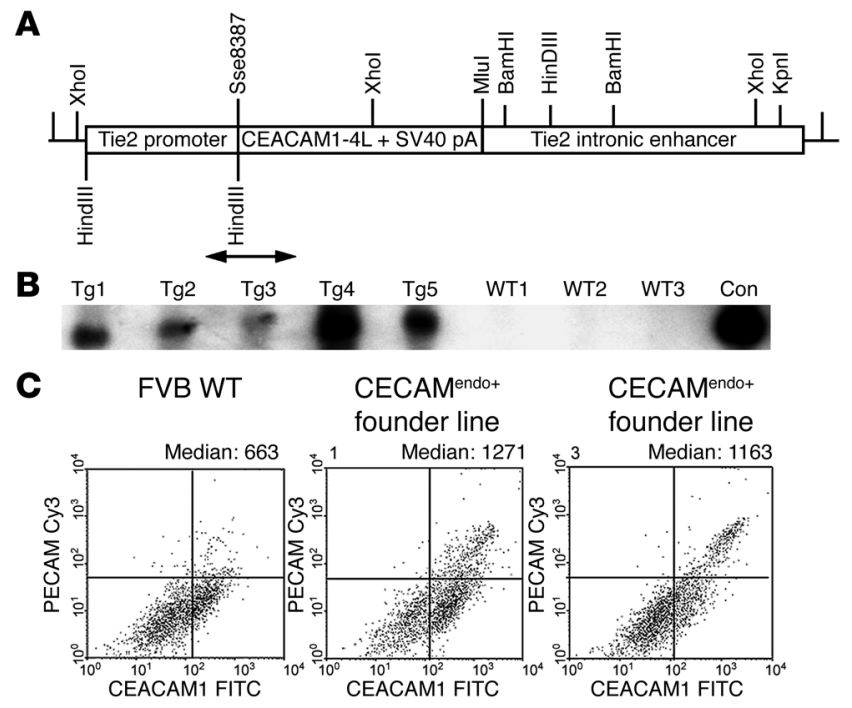


A
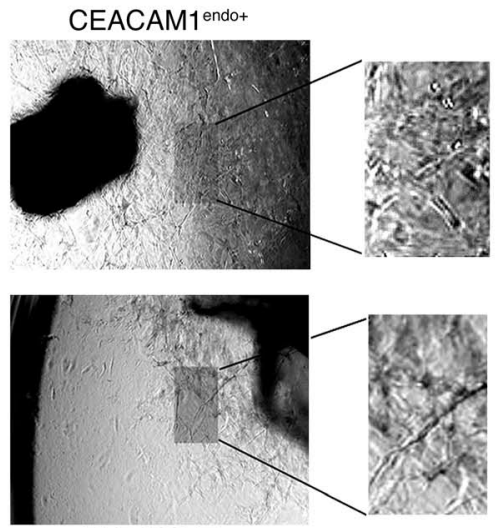

FVB/N WT

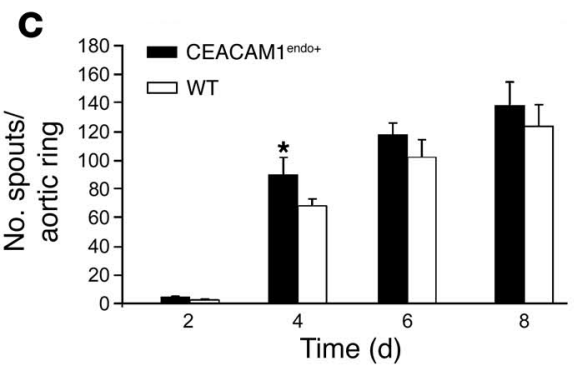

Ceacam1--
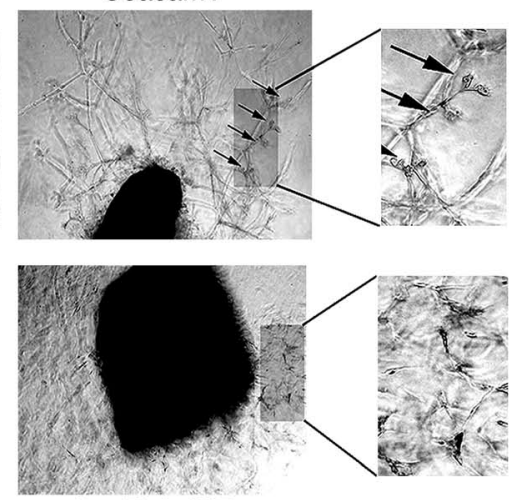

C57BL/6 WT

D

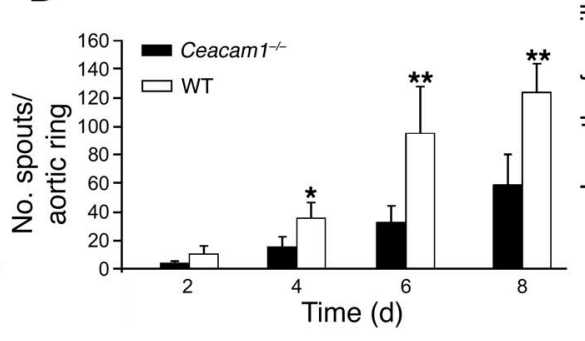

B

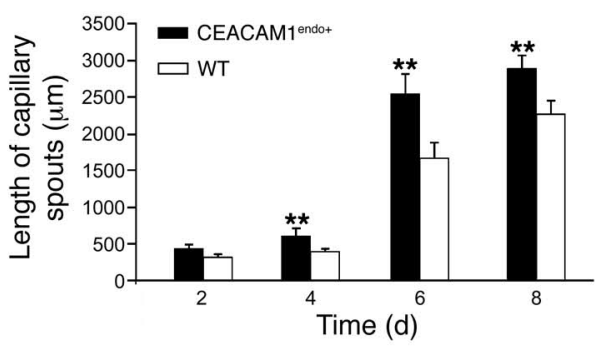

E

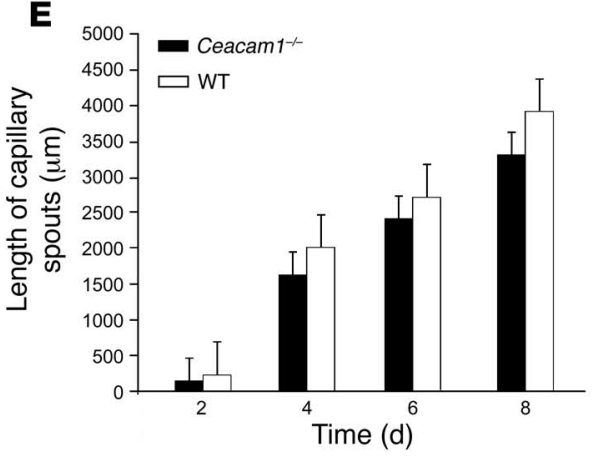

Figure 3

Aortic ring assays of CEACAM1 endo+ and Ceacam1-/- mice. (A) Aortic ring assays of Ceacam1-/- mice, CEACAM1endo+ transgenic animals, and their WT littermates. Aortas were cut into 1-mm-thick rings, implanted into Matrigel, and allowed to grow for 10 days. Images were taken on day 8 , and endothelial cell tube formation and tubular branching into capillary-like networks were compared. In Ceacam $1^{-1-}$ mice, the capillary network exhibited lower complexity compared with the branched pseudovascular trees in CEACAM1endo+ mice, FVB/N WT, and C57BL/6 WT mice. Arrows show the lack of connection among neighboring protrusions. Magnification, $\times 100, \times 200$ (insets). (B and C) Aortic ring assays were performed from at least 6 CEACAM1 endo+ and WT littermates, and the overall length of endothelial cell sprouts (B) and the overall number of endothelial cell sprouts emerging from the aortic ring $(\mathbf{C})$ were determined. The invasion length of capillary tubes formed by CEACAM1endo+ significantly exceeded that of their WT littermates on days 4, 6, and 8 . Significant differences in numbers of endothelial cell tubes were obtained on day 4 of the assay. ${ }^{*} P<0.05 ;{ }^{* *} P<0.01$. (D and $\mathbf{E}$ ) Aortic ring assays were performed from at least 6 Ceacam $1^{-/-}$and WT littermates, and the number of endothelial cell sprouts per aortic ring (D) and the overall length of capillary protrusions in aortic explants (E) were determined. The number of endothelial protrusions per aortic ring in Ceacam $1^{-1-}$ mice was significantly different compared with WT mice on days 4,6 , and 8 . No significant differences in the length of the endothelial pseudotubes were detected.

Ser $_{503}$ residues - or both - were no longer capable of invading a 3-dimensional ECM and differentiating into capillary-like networks. Instead, they formed single-cell clusters or colonies (Figure 1B). In agreement with previously published reports, these data provide further evidence that expression of CEACAM1 with an intact long cytoplasmic domain regulates cellular motility, invasion, and differentiation $(24,25,28)$.

Generation of an animal model for CEACAM1 function in angiogenesis. For evaluation of the impact of CEACAM1 in angiogenesis in vivo, we used 2 different murine models. We generated CEACAM1 ${ }^{\text {endo+ }}$ mice on an $\mathrm{FVB} / \mathrm{N}$ background with additional CEACAM1-L expression under the endothelial cell-specific promoter control of the Tie 2 receptor tyrosine kinase. In order to observe the functional consequences of endothelial CEACAM1 deficiency, we also used Ceacam 1 $1^{-/}$mice with systemic deletion of the Ceacam 1 gene (29). For the CEACAM1 $1^{\text {endo+ }}$ transgenic line, we modified a construct by T.N. Sato (30), replacing the $\beta$-galactosidase reporter gene ( $\mathrm{Lac} Z$ ) by that of the Ceacam1-L cDNA, as shown in Figure 2A. Transgenic founder lines were identified by Southern blotting (Figure 2B), and 2 transgenic lines were used in the experiments described here. We also used 2 independent lines of the Ceacam1-/- mice in our experiments. Transgenic and knockout mice were genotyped by PCR (data not shown). To verify CEACAM1 overexpression in the endothelia of CEACAM $1^{\text {endo+ }}$ transgenic animals, we double labeled primary endothelial cells isolated from lungs with anti-PECAM1 and anti-CEACAM1 antibodies in flow cytometry (Figure 2C). Primary endothelial cells from CEACAM $1^{\text {endo+ }}$ mice exhibited overexpression, revealed by both CEACAM1 and PECAM1 staining, compared with their corresponding WT siblings. We also confirmed CEACAM1 overexpression of endothelial cells in adult animals by Western blotting and RT-PCR (data not shown). Macroscopically, no overt vascular damage or alterations were observed under physiological conditions in Ceacam $1^{-/}$or CEACAM1 $1^{\text {endo+ }}$ transgenic mice. The animals were viable and fertile.

Endothelial CEACAM1-L expression enhances endothelial cell outgrowth. To compare the angiogenic properties of endothelial cells of Ceacam $1^{-/-}$and CEACAM1 ${ }^{\text {endo+ }}$ transgenic mice, we prepared aortas from transgenic mice and their appropriate WT siblings and performed aortic ring assays (31). Expression of PECAM1 and CEACAM1 in aortic endothelia was validated prior to these experiments by immunohistochemical analyses (data not 
CEACAM $1^{\text {endo+ }}$

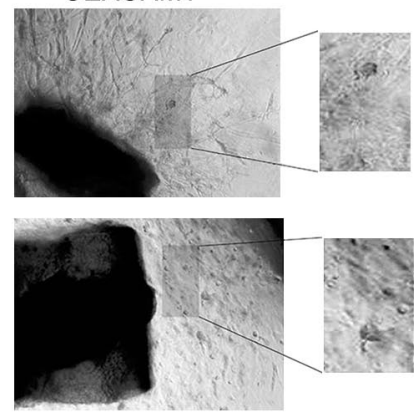

FBV/N WT

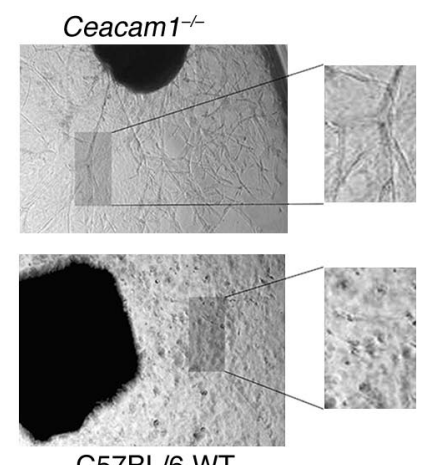

C57BL/6 WT
Figure 4

Destabilization of endothelial cell networks by anti-CEACAM1 antibodies. Anti-CEACAM1 antibody AgB10 $(0.7 \mu \mathrm{M})$ was added to explants of Ceacam $1^{-1-}$ mice, CEACAM1 ${ }^{\text {endo+ }}$ mice, and their appropriate WT littermates for 6 days, and interference with endothelial tube formation is shown. Representative areas of the explants are marked with gray boxes and enlarged correspondingly on the right side of each panel. Magnification, $\times 100, \times 200$ (insets).

shown). Luminal CEACAM1 expression was found in CEACA$\mathrm{M}^{\text {endo+ }^{\text {en }}}$ mice, whereas no CEACAM1 expression was detected in Ceacam $1^{-1-}$ mice (data not shown). This is different from the human expression pattern, where CEACAM1 can only be detected in small capillaries and newly formed vessels (12). All genetically unaltered animals exhibited CEACAM1 expression in their aortic endothelium. No differences in aortic endothelial PECAM1 expression were observed in CEACAM $1^{\text {endo+ }}$ or Ceacam $1^{-/-}$mice or their WT littermates (data not shown).

Results from the aortic ring assays, analyzing the newly formed endothelial cell network protruding from aortic explants, are compared in Figure 3A. Endothelial cell tubes protruding from aortic explants of Ceacam $1^{-/-}$mice formed a less complex endothelial network compared with the rather dense network emerging from explants of their WT littermates. Instead, buds formed at endothelial branching sites of the endothelial network in the Ceacam 1-/- aortic explants did not sprout or develop into pseudocapillary tubes. Additionally, they did not interconnect with neighboring protrusions (Figure $3 \mathrm{~A}$, arrows). However, no morphological differences in aortic endothelial cell outgrowth and invasion between WT and heterozygous Ceacam $1^{+/-}$mice were observed (data not shown). Concurrent with the hypothesis that CEACAM1 supports endothelial cell outgrowth in early stages, aortic endothelial cell invasion and branching were more pronounced in aortic explants from CEACAM $1^{\text {endo+ }}$ mice compared with specimens from their WT littermates (Figure 3A). Here the overall invasive capacity of endothelial cells protruding from the explants was more pronounced in CEACAM $1^{\text {endo+ }^{+}}$mice, as calculated by measuring the overall length of the endothelial branches (Figure 3B). On the other hand, the overall number of protrusions emanating from the aortic explants only differed significantly during early phases of endothelial cell outgrowth (Figure 3C). In marked contrast to the CEACAM1 $1^{\text {endo+ }}$ mice, the Ceacam 1 $1^{-/}$mice developed a dramatically smaller number of endothelial protrusions compared with their WT littermates (Figure 3D). Differences in the length of endothelial sprouts between Ceacam 1 ${ }^{-1-}$ mice and their WT littermates were observed but were not significant (Figure 3E).
Anti-CEACAM1 antibodies destabilize endothelial cell networks. As described above, the aorticendothelium of FVB/NWT, CEACAM1 ${ }^{\text {endo+, }}$, and C57BL/ 6 WT mice expressed the CEACAM1 protein. To verify CEACAM1-dependent effects on endothelial cell outgrowth of aortic explants, we supplemented the growth media with the purified monoclonal anti-CEACAM1 antibody AgB10. Figure 4 shows the summary of these inhibition experiments, with representative areas of the newly formed endothelial cell networks shown at higher magnification. After application of the anti-CEACAM1 antibody $\mathrm{AgB} 10(0.7 \mu \mathrm{M})$, the tubular structures emerging from the aortic explants of Ceacam $1^{-/-}$mice remained morphologically intact. This is in agreement with the observation that CEACAM1 is not expressed on the luminal endothelia of Ceacam1 1/- mice. In sharp contrast, application of AgB10 to the aortic explants of C57BL/6 WT mice induced a morphological disintegration of newly formed capillaries and the formation of isolated cell clusters. This result was also observed in AgB10-treated explants of $\mathrm{CEACAM}^{\mathrm{endo}^{+}}$and FVB/N WT mice. Using isotype-matched controls, the overall architecture of the network was not affected in the CEACAM1 $1^{\text {endo+ }}$ or Ceacam $1^{-/-}$mice or their appropriate WT littermates (data not shown).

CEACAM1 $1^{\text {endo+ }}$ transgenic mice exhibit increased neovascularization, whereas neovascularization in Ceacam $1^{-/-}$mice is diminished. To further assess the angiogenic potential of CEACAM1 ${ }^{\text {endo+ }}$ and Ceacam 1 $1^{-/}$mice, we used a Matrigel plug assay: Matrigel was subcutaneously injected into the mice, and the implants were removed after 3 weeks to evaluate neovascularization after histological processing. To allow efficient vascularization, angiogenic growth factors angiopoietin-1 (Ang-1), bFGF, and VEGF were suspended in the Matrigel prior to injection. Controls did not contain growth factor supplements. Figure 5A shows histological analysis of Matrigel plugs containing Ang-1 retrieved from CEACAM $1^{\text {endo+ }}$ and Ceacam $1^{-/-}$mice and their corresponding WT siblings. Newly formed vessels were positive for CEACAM1 (blue; counterstain, Nuclear Fast Red) and PECAM1 (red; counterstain, hematoxylin) in CEACAM $1^{\text {endo+ }}$ transgenic mice and their WT littermates. In plugs from the Ceacam $1^{-/-}$mice (counterstain, hematoxylin), no CEACAM1 staining could be detected. Strikingly, extravascular erythrocytes were detected in the proximity of vessel-like structures in the implants of Ceacam $1^{-/-}$mice (Figure 5A, arrows). However, cell populations in the Matrigel implants from Ceacam $1^{-/}$mice showed no reactivity toward anti-PECAM1 or anti-von Willebrand factor antibodies (data not shown). In the appropriate WT controls, both CEACAM1 and PECAM1 expression was revealed in newly formed capillaries in the implants.

To confirm that CEACAM1-positive capillaries are also positive for PECAM1, we performed immunofluorescent double labeling of CEACAM1 and PECAM1 in Matrigel explants from the CEACAM1 $1^{\text {endo+ }}$ mice. Figure 5B shows double labeling of CEACAM1 (green fluorescence) and PECAM1 (red fluorescence) as well as the overlay (yellow), in which congruent expression of endothelial CEACAM1 and PECAM1 was demonstrated.

Statistical analyses of the overall vascular densities in Matrigel plugs are shown in Figure 5, C and D. CEACAM1 $1^{\text {endo+ }}$ transgenic animals exhibited higher neovascularization of the Matrigel implants upon angiogenic challenge with Ang-1, bFGF, and VEGF compared with their WT littermates (Figure 5C). Contrary to these findings, the Ceacam $1^{-/-}$mice exhibited significantly reduced neovascularization of the implants, and only very few intact vessels 
A

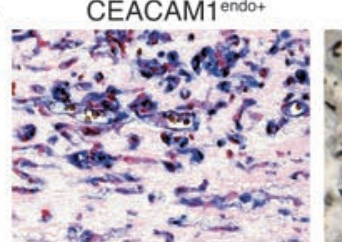
Anti-CEACAM1

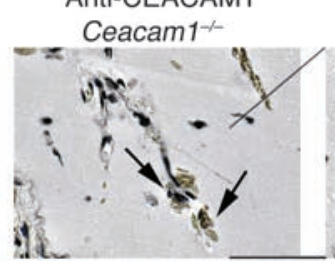

Hematoxylin

WT (FVB/N)

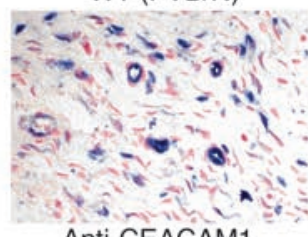

Anti-CEACAM1

WT (C57BL/6)

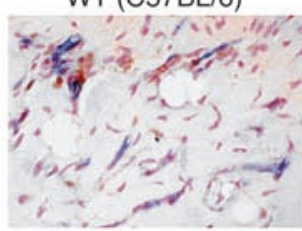

Anti-CEACAM1

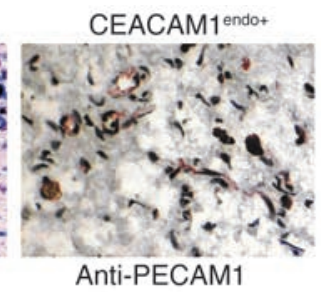

Anti-PECAM1

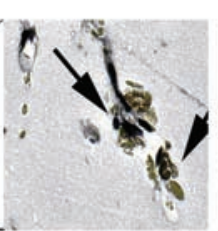

WT (FVB/N)

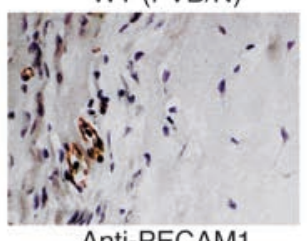

Anti-PECAM1

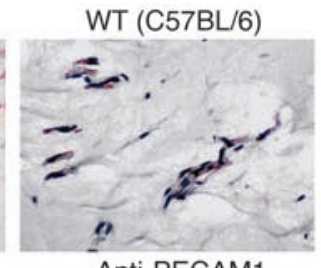

WT (C57BL/6)

Anti-PECAM1

B

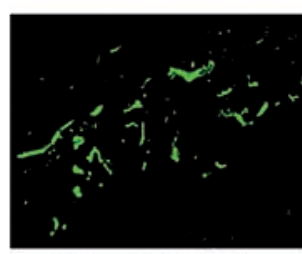

Anti-CEACAM1

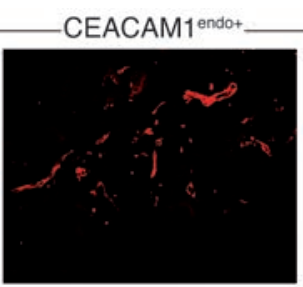

Anti-PECAM1

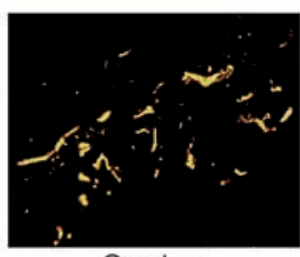

Overlay

C

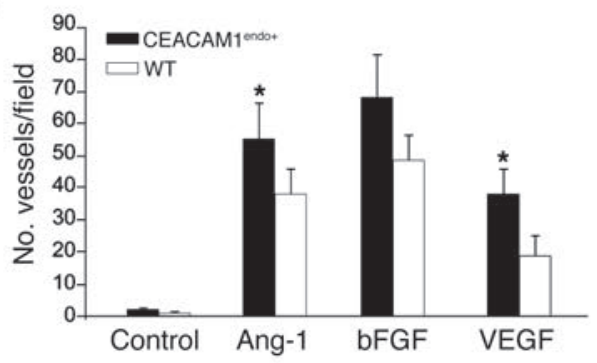

D

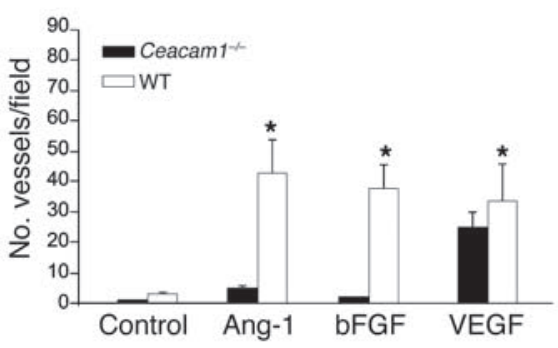

Figure 5

Qualitative and quantitative assessment of newly formed vessels in Matrigel implants. (A) Representative histological paraffin sections of Matrigel plugs. In CEACAM1 ${ }^{\text {endo+ }}$ mice, newly formed vessels in the plug area were CEACAM1 positive (anti-CEACAM1 antiserum 2456, blue; counterstain, Nuclear Fast Red) and PECAM1-positive (red; counterstain, Mayer's hemalaun). Plugs shown here contained recombinant Ang-1. In plugs from Ceacam1-- mice (counterstain, Mayer's hemalaun), the majority of CEACAM1-negative vessel-like structures in Matrigel plugs were defective: extravascular erythrocytes were observed (arrows). This area is enlarged at right. Implants from the corresponding WT siblings of CEACAM1 endo+ and Ceacam 1-l- mice are also shown. Newly formed vessels revealed reactivity toward CEACAM1 and PECAM1 antibodies. Magnification, $\times 200, \times 400$ (inset). (B) Immunofluorescent labeling of CEACAM1 and PECAM1 in newly formed capillaries in a Matrigel plug from a CEACAM1 endo+ mouse. Congruent endothelial expression of CEACAM1 (green, anti-CEACAM1 antiserum 2456) and PECAM1 (red, anti-PECAM1 antibody BM4086) is shown in the overlay. Magnification, $\times 200$. (C) Neovascularization in Matrigel plugs from CEACAM1endo+ mice and their FVB/N WT siblings. After angiogenic challenge with Ang-1, bFGF, and VEGF, PECAM1-positive vessels were counted per field. A total of 3 fields from individual plugs of 3 animals per group were counted. (D) Quantitative comparison of vascularization in Matrigel implants from Ceacam1-/- mice and their WT littermates. In Ceacam1-/- mice, vessel-like structures associated with erythrocytes were also considered. Significant differences in the number of vessels formed in Matrigel implants in CEACAM1 ${ }^{\text {endo+ }}$ and Ceacam $1^{-1-}$ mice compared with their WT littermates are indicated by asterisks $\left({ }^{\star} P<0.05\right)$.

were detected, whereas the C57BL/6 WT mice responded to angiogenic stimuli appropriately (Figure 5D). Without the addition of extra supplements to trigger neoangiogenesis (i.e., controls), the implants remained largely avascular.

Based on our observations that Ceacam $1^{-/-}$mice exhibited structures within their Matrigel implants that were reminiscent of defective vessels and the finding that the number of intact vessels was dramatically lower compared with specimens from C57BL/6 WT littermates, we sought to determine whether the presence of extravascular red blood cells was associated with actual vascular leakage induced by vascular growth factors. For this purpose, we injected FITC-labeled dextran into the tail veins of the mice prior to removal of the implants for general vascu- lar labeling. Figure 6 presents results obtained after histological processing of the Matrigel plugs containing VEGF. The effects of VEGF on the neovascularization in Ceacam $1^{-/-}$mice and a WT sibling are shown in representative areas of the Matrigel implants: in the implant removed from a Ceacam $1^{-/-}$mouse, diffuse smears of fluorescein were detected (Figure 6, white arrows), whereas rather well-defined fluorescent structures were visible after i.v. injection of FITC-labeled dextran into WT animals, and only moderate penetration of FITC-labeled dextran through newly formed vessels was observed in WT mice (Figure 6, yellow arrows). In Matrigel implants without any supplements, very few intact vessels were detected in the Ceacam $1^{-/-}$mice and the C57BL/6 mice (data not shown). To elucidate whether 


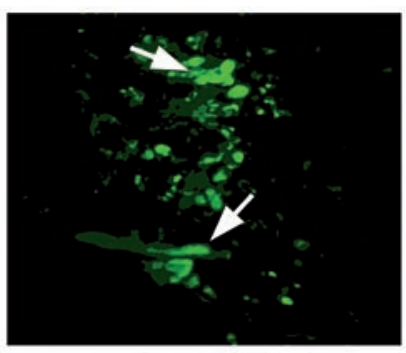

Ceacam $1^{-/-}$

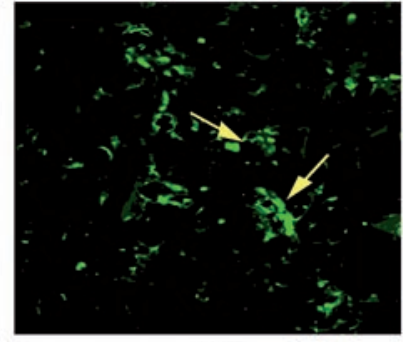

WT

\section{Figure 6}

Visualization of FITC-labeled dextran in VEGF-treated Matrigel plugs retrieved from Ceacam $1^{-1-}$ mice and their WT littermates after tail vein injection. Vascular distribution of FITC-labeled dextran was visualized by fluorescence microscopy. White and yellow arrows indicate diffuse distribution and vascular penetration of FITC-labeled dextran, respectively. Magnification, $\times 200$.

these differences between Ceacam 1/-/- mice and their WT siblings were based on specific morphological features of the vascular architecture, we subjected the Matrigel implants to analyses by electron microscopy. In Figure 7, examples of these ultrastructural analyses of VEGF-soaked Matrigel plugs are given: In the CEACAM1 ${ }^{\text {endo+ }}$ mouse (Figure 7, left panel), an intact vessel is shown, consisting of the cross-section of an endothelial cell, a pericyte, deposits of ECM, and a basal lamina (thick arrows). In the Ceacam $1^{-/-}$mice (Figure 7, right panels), we surprisingly did not detect any intact vascular structures. Instead, apoptotic cells were present (arrowheads) as well as red blood cells that were not enclosed by capillaries but were randomly distributed within poorly organized deposits of collagen (thin arrows), and no architectural hallmarks of an orderly vascular endothelial structure were detected.

Ceacam $1^{-1-}$ mice show significantly reduced collateral blood flow after bind limb ischemia in contrast to CEACAM1 $1^{\text {endo+ }}$ mice. To validate a functional role for CEACAM1 in vascular remodeling in vivo, we investigated vascular growth after induction of ischemia via unilateral femoral artery occlusion in CEACAM1 ${ }^{\text {endo+ }}$ and Ceacam $1^{-/-}$ mice and their respective WT littermates. These experiments were based on the previous observation that hypoxia induces CEACAM1 expression in synergy with other angiogenic factors in ischemic cardiac muscle (19).

As a functional parameter, we determined collateral blood flow 1 week after femoral artery occlusion via fluorescent microspheres employing established methods of blood flow determination in mice (32). In addition, we determined maximum systolic blood flow via a transonic flow probe placed at the collateral stem region as an indirect parameter of collateral conductance. Finally, we determined vascular growth by assessing capillary and arteriolar densities in the different calf muscles. The ischemic hind limb model offers the advantage that vascular growth is investigated in a physiologically relevant setting. Perfusion recovery in the ischemic leg was expressed as percent perfusion of the nonligated leg as described previously (32).

These data are summarized in Figure 8 and Table 1. Equal perfusion of both kidneys served as reference for adequate microsphere mixing and injection (data not shown) $(32,33)$. In agreement with the results obtained in neovascularization of Matrigel plugs, the relative perfusion of ischemic versus nonischemic limbs 7 days after surgery was significantly higher in CEACAM1 ${ }^{\text {endo+ }}$ mice compared with their WT littermates (Figure 8 and Table 1; for CEACAM1 $1^{\text {endo+ }}$ mice, $75 \% \pm 5 \%$; for WT littermates, $54 \% \pm 6 \% ; P<0.05$ ). Perfusion in ischemic hind limbs in Ceacam 1-/- mice was significantly lower than their genetically unaltered siblings (Ceacam $1^{-/-}$mice, $45 \% \pm 3 \%$; WT mice, $62 \% \pm 3 \%$; $P<0.01)$. Maximal blood flow amplitudes as determined by transonic flow probes also showed a clear tendency toward higher blood flows in CEACAM $1^{\text {endo+ }}$ mice in the ischemic hind limb compared with Ceacam $1^{-/-}$animals (Table 1). These differences, however, did not reach statistical significance, due to large standard deviations. The alteration in blood flow recovery between the different WT groups is in accordance with previous studies showing a marked variation in the arteriogenic response among different mouse strains (34). The lower perfusion rates in Ceacam1 $1^{-1-}$ mice correlated with different vascular densities in ischemic calf muscles after femoral artery ligation - as demonstrated in Figure 9, immunofluorescent labeling of PECAM1 showed that vascular densities were markedly increased in CEACAM $1^{\text {endo+ }}$ compared with Ceacam $1^{-/-}$mice.

\section{Discussion}

Our previous in vitro data suggested that CEACAM1 is involved in angiogenesis. This is supported by a recent proteomic screen for cell membrane components expressed in newly formed tumor vessels and the fact that CEACAM1 expression is upregulated in synergy with other angiogenic factors in cardiac hypoxia $(17,19)$. To date, however, evidence for a causal implication of CEACAM1 in angiogenesis in vivo was lacking. In the present study, we report on 2 different genetic mouse models in which the angiogenic action of CEACAM1 has been investigated: in CEACAM1 $1^{\text {endo+ }}$ mice, the expression of CEACAM1-L was targeted to endothelia via the Tie 2 promoter, and in Ceacam $1^{-/}$mice, the Ceacam 1 gene was inactivated by targeted disruption (29). In addition, endothelial cells were transfected with cDNAs coding for WT CEACAM1-L and for CEACAM1-L mutants harboring amino acid substitutions in the cytoplasmic domain. In these experimental systems, we provide conclusive

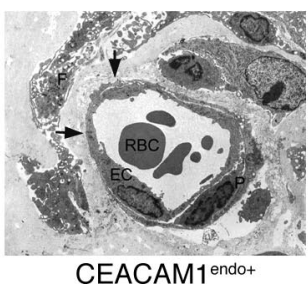

\section{Figure 7}

Electron microscopy of Matrigel implants. To analyze the underlying vascular defects in Matrigel implants from Ceacam $1^{-/-}$mice and to verify vessel integrity in CEACAM1 endo+ mice, the implants were subjected to electron microscopy. Here we chose implants that had been treated with VEGF. In specimens from CEACAM1 ${ }^{\text {endo+ }}$ mice, an intact capillary consisting of an endothelial cell (EC) and a pericyte $(P)$ as well as a basal lamina (thick arrows) was detected. Strikingly, no capillaries were present in explants from Ceacam $1^{-/-}$mice; instead, apoptotic cells (arrowheads) and extravascular red blood cells (RBC; thin arrows) were visible. In addition, poorly organized bundles of collagen (C) were scattered within the explants. Magnification, $\times 2,250$. 
A

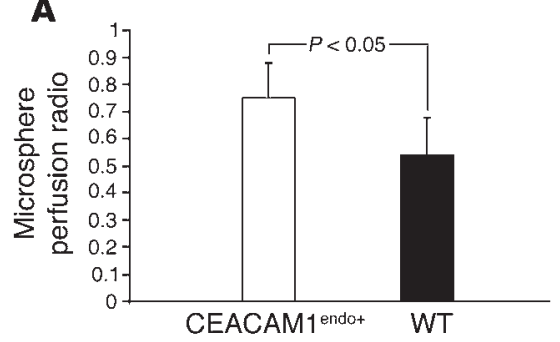

B

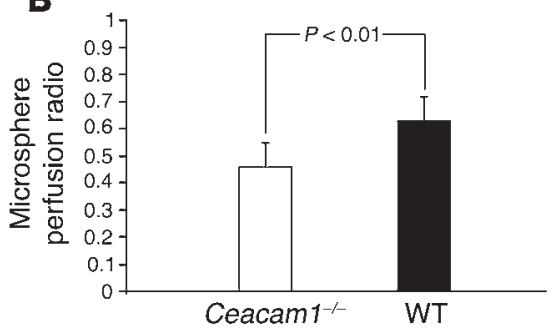

Figure 8

Microsphere perfusion ratios. Perfusion after ischemia was assessed via relative microsphere perfusion ratios, expressed as a ratio of microsphere counts per gastrocnemial muscle of ligated over nonligated leg. Shown are results obtained in CEACAM1 endo+ mice and their WT siblings (A; $n=5$ per group) and Ceacam $1^{-/}$ mice and their WT controls (B; $n=6$ per group). Bars indicate SD. evidence that CEACAM1 is involved in angiogenesis and vascular remodeling. (a) CEACAM1 expression enhanced the invasive growth of endothelial cells into ECM matrices. Both stable endothelial CEACAM1 transfectants and endothelial cells from aortas of transgenic mice exhibited increased invasion of ECM matrices. In CEACAM1 transfectants, substitution of $\mathrm{Tyr}_{488}$ or $\mathrm{Ser}_{503}$ in the cytoplasmic domain of CEACAM1-L abrogated the CEACAM1-dependent invasive growth properties. (b) CEACAM1 affected morphogenesis of endothelial cells and blood vessels. In fibrin gels, CEACAM1-transfected endothelial cells differentiated into tubelike structures. In aortic ring assays, branching of newly formed sprouts was more pronounced in endothelial cells from transgenic animals compared with their WT littermates. On the contrary, in aortic explants from the Ceacam $1^{-/-}$ mice, endothelial tube formation was impaired. The formation of tubular networks by CEACAM1-expressing endothelial cells from CEACAM1 $1^{\text {endo+ }}$ transgenic and WT animals was inhibited by a monoclonal anti-CEACAM1 antibody, whereas the antibody did not interfere with the growth of endothelial tubes from Ceacam $1^{-/-}$mice. (c) CEACAM1 affected the neovascularization of subcutaneous Matrigel implants. When Matrigel plugs containing angiogenic growth factors such as VEGF, bFGF, or Ang-1 were transplanted subcutaneously, an increased number of blood vessels was observed in CEACAM $1^{\text {endo+ }}$ mice, whereas only few intact capillaries penetrated the implants in the Ceacam 1-/- mice. (d) CEACAM1 expression was essential for vascular remodeling and vessel stabilization in vitro and in vivo. In Matrigel plugs containing Ang-1 or VEGF from Ceacam $1^{-/-}$mice, perturbation of vascular integrity was observed through the presence of extravascular FITC-labeled dextran and red blood cells. This decrease in vascular stability was confirmed by ultrastructural analyses of the Matrigel implants, which showed no intact capillaries in specimens from Ceacam $1^{-/-}$mice, but rather apoptotic cells and extravascular erythrocytes scattered within deposits of collagen. Hence we suggest that CEACAM1 supports angiogenesis and vascular remodeling by stabilization of nascent vessels. Moreover, cell populations in the Matrigel plugs from Ceacam $1^{-/-}$mice appeared to be rather uniform, as interactions with accessory cells, such as pericytes and fibroblasts, were largely absent. (e) A functional role for CEACAM1 in vascular remodeling was also confirmed in our in vivo experiments, after the induction of hypoxia by ligation of the femoral artery: CEACAM $1^{\text {endo+ }}$ mice exhibited significantly higher perfusion in their ischemic calf muscles compared with their WT littermates, and collateral vessel growth was perturbed in Ceacam 1-/- mice as demonstrated by significantly lower rates of perfusion compared with WT controls.

Notably, normal vasculogenesis appears not to be affected in the Ceacam $1^{-/}$mice, since no obvious vascular defects are observed and the animals are fertile. This finding may be explained by the fact that mice possess an additional Ceacam gene (encoding the CEACAM2 protein) that may compensate for the function of CEACAM1, although the role of this protein in angiogenesis has not yet been investigated (35).

In general, members of the IgSF of adhesion molecules like CEACAM1 exhibit angiogenic effects in adults but appear to be dispensable for vasculogenesis in the embryo. Anti-PECAM1 antibodies affect the growth of blood vessels into a subcutaneously implanted ECM matrix and reduce tumor angiogenesis, though PECAM1-deficient mice show no overt vascular defects $(8,9,36)$. Similarly, targeted disruption of the endothelial cell-selective adhesion molecule reduces the vascularization of Matrigel plugs and reduces growth of transplanted tumors, whereas vasculogenesis remains unaffected (10). This is different from the role of other families of adhesion molecules, such as cadherins and integrins, in which targeted disruption of the respective genes leads to defects in vasculogenesis. For example, disruption of the VE-cadherin gene results in embryonic lethality due to vascular defects (37). Similarly, ablation of $\alpha_{5}$ integrin leads to defective mesoderm formation and embryonic lethality (38).

In contrast to other members of the IgSF of cell adhesion molecules with a direct functional implication in vascular remodeling in vivo in response to hypoxia, we show here that abrogation of CEACAM1 expression in Ceacam $1^{-/-}$mice resulted in deficiencies in endothelial cell assembly into new vessels and vascular remodeling. This was demonstrated by supplementing Matrigel implants in the Ceacam 1-/- mice with either Ang-1 or VEGF. Ang-1 has been implicated in vessel remodeling, maturation, and stabilization, and VEGF, formerly designated vascular permeability factor (VPF), induces endothelial cell proliferation and regulates vascular permeability as well as the initial assembly of vessels during vasculogenesis and vessel expansion during angiogenesis $(4,39)$.

So far, the molecular basis of the angiogenic action of CEACAM1 is largely unresolved. Since CEACAM1 is a homophilic cell adhesion molecule, CEACAM1 may mediate endothelial

\section{Table 1}

Summary of results obtained after unilateral femoral artery ligation in CEACAM1 $1^{\text {endo+ }}$ and Ceacam $1^{-/-}$mice

\begin{tabular}{|c|c|c|c|c|}
\hline & Ceacam1-/- & $\begin{array}{c}\text { WT } \\
\text { (C57BL/6) }\end{array}$ & CEACAM1 $1^{\text {endo+ }}$ & $\begin{array}{c}\text { WT } \\
\text { (FVB/N) }\end{array}$ \\
\hline Perfusion ratio (\%) & $45 \pm 3^{A}$ & $62 \pm 3^{A}$ & $75 \pm 5^{\mathrm{B}}$ & $54 \pm 6^{B}$ \\
\hline MBFA [ul] & $138 \pm 22$ & $270 \pm 113$ & $180 \pm 83$ & $138 \pm 22$ \\
\hline
\end{tabular}

Collateral flows are expressed as occluded to nonoccluded hind limb perfusion ratios and maximum blood flow amplitude (MBFA) to the ischemic leg. ${ }^{A} P<0.01$. ${ }^{B} P<0.05$. 


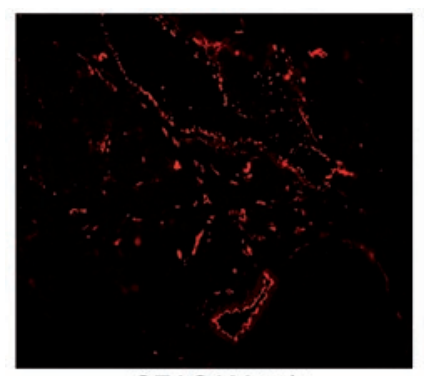

CEACAM $1^{\text {endot }}$

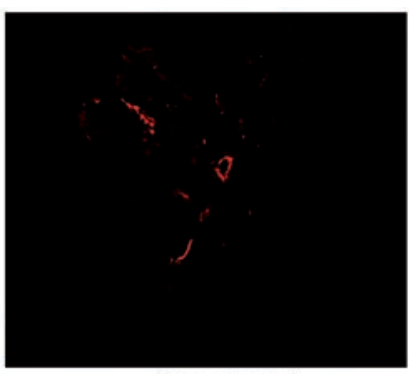

Ceacam $1^{-1-}$

cell-cell interactions. In addition, recent reports indicate that CEACAM1 interacts with integrins and receptor tyrosine kinases in cis. CEACAM1 colocalizes with $\beta_{3}$ integrin at the invasive front of melanomas and in the human intermediate trophoblast (23, 24). According to our own previous observations, interaction of CEACAM1 with $\beta_{3}$ integrin requires tyrosine phosphorylation of the CEACAM1-L cytoplasmic domain. In human melanoma and melanocytic cells, CEACAM1-L-dependent migration and invasive growth can be blocked by compounds that interfere with integrin-mediated adhesion (25). In agreement with the results reported here, replacement of $\mathrm{Tyr}_{488}$ reduced the CEACAM1dependent increase in migration and invasion (25). Interestingly, integrin $\alpha_{v} \beta_{3}$ is also involved in the regulation of angiogenesis and progression of malignant tumors (40-43). Taken together, these findings indicate that CEACAM1 and integrins are functionally interconnected. According to results from Najjar and coworkers, CEACAM1 is tyrosine phosphorylated after ligand binding to the insulin and EGF receptors. In turn, the activity of these growth factor receptors is modulated by CEACAM1 $(26,27)$. Similar to these mechanisms, CEACAM1 may exert its angiogenic properties by regulating the activity of integrins and receptor tyrosine kinases in endothelial cells. In addition to these potential activities, CEACAM1 expression has been reported to upregulate angiogenic growth factors such as VEGF, angiogenin, and angiopoietins but is also upregulated itself under hypoxic conditions or by VEGF $(16,19)$.

Taken together, our findings provide evidence that CEACAM1 is involved in the invasion of endothelial cells into the ECM as well as in the regulation of capillary sprouting and remodeling into more mature vessels. In addition, the perturbed vascular stabilization observed in CEACAM1-deficient animals in response to angiogenic growth factors or hypoxia points to an essential role for CEACAM1 in vascular growth. Thus it is possible that clinical alteration of ligand binding or cell signaling by CEACAM1 may offer a novel strategy for modulation of angiogenic processes in the treatment of disease.

\section{Methods}

Generation of stable CEACAM1-expressing SVEC4-10 transfectant cells. Stable CEACAM1 transfectant cells were generated with the murine endothelial cell line SVEC4-10 (ATCC CRL-2181) by retroviral infection. CEACAM1-4L and CEACAM1-4L mutants (Y488F, S503A, and Y488F;S503A) were generated by site-directed mutagenesis $(44,45)$. The Y488F;S503A mutant was generated by overlap PCR using primers AH1 (5'-GACGTCGCATTCACTGTCCTGAACTTCAATTCCCAGCAACCCAACCGGCCAACTGCAGCCCCTTC$\left.3^{\prime}\right)$ and NBIT2 (5'-CATCACTGGTGAATTCAGCAGGAC-3') (46). Three independent cell clones were selected with $\mathrm{G} 418(1 \mathrm{mg} / \mathrm{ml})$ by limiting dilution and used in the experiments described herein.

\section{Figure 9}

Immunofluoresecent labeling of capillaries with anti-PECAM1 antibodies in ligated, ischemic calf muscles. Representative cryostat sections of specimens from CEACAM1 $1^{\text {endo+ }}$ and Ceacam $1^{-/-}$mice after femoral artery ligation were compared. Note that very few capillaries were present in the ischemic calf muscles of Ceacam $1^{-1-}$ mice. For capillary labeling, anti-PECAM1 antibody BM4086 was used. Magnification, $\times 200$.

Transwell assays. Invasion assays were performed in a transwell system (BD Biosciences) with Matrigel-coated filters, and cellular invasion was evaluated after 6 hours (25).

Fibrin assays. Fibrin assays were performed with single cell suspensions as previously described (47). Microscopic evaluations in this report were performed with a Zeiss Axioplan microscope.

Generation of transgenic mice. The lacZ cassette of the pHHNS vector (T.N. Sato, University of Texas Southwestern Medical Center, Dallas, Texas, USA; ref. 30) was exchanged for the murine Ceacam1-4L cDNA followed by a SV40 polyadenylation signal (44). The construct was subjected to DNA sequencing prior to use. Transgenic mice were generated by microinjection into $\mathrm{FVB} / \mathrm{N}$ mouse oocytes. $\mathrm{FVB} / \mathrm{N}$ mice were obtained from Harlan. Care of the mice was taken according to standards defined by the Canadian Council on Animal Care and section 8 of the German Law for the Protection of Animals.

Ceacam 1-/- mice. Ceacam $1^{-/-}$mice were generated by N. Beauchemin as described previously (29).

Genotyping of mice. Ceacam 1-/- mice were genotyped by PCR (29). The CEACAM $1^{\text {endo+ }}$ transgenic mice were characterized by Southern blotting after EcoRI restriction digest of genomic DNA from tail biopsies or by PCR. In both cases, an overlapping fragment of the Tie2 promoter and the $\mathrm{N}$-terminal CEACAM1 domain was used as a probe or as a target for amplification by PCR using primers 2 T5 (5'-GGGAAGTCGCAAAGTTGTGAGTT-3') and 46N1 (5'-CTTCATGGTGATTTTGG-3').

Isolation of primary endothelial cells from lung. Primary endothelial cells from murine lungs were isolated as described previously (48).

Flow cytometric analyses. Double labeling of CEACAM1 and PECAM1 on primary endothelial cells was performed as described previously (30). For CEACAM1, the monoclonal anti-CEACAM1 antibody AgB10 (a kind gift from T. Rudinskaya and G. Abelev, Institute of Carcinogenesis, N.N. Blokhin Russian Cancer Center Research Center, Moscow, Russia) was used.

Ex vivo aortic ring assays. Aortas were prepared from 8- to 10-week-old mice, and aortic ring assays were performed as previously reported (31). For inhibition studies, the anti-CEACAM1 antibody AgB10 was used $(10 \mu \mathrm{g} / \mathrm{ml})$. Endothelial cell outgrowth was monitored for 10 days. Statistics were performed starting at day 2. For quantification, endothelial cell sprouts were counted and their total length was measured.

In vivo Matrigel plug assays. Mice were injected subcutaneously with $0.5 \mathrm{ml}$ Matrigel (BD Biosciences) containing either 200 ng recombinant Ang-1, 120 ng recombinant murine VEGF ${ }^{120}$, or 120 ng bovine bFGF (all R\&D Systems) as described previously (49). Controls did not contain any growth factors. Neovascularization in Matrigel implants was gauged after tail vein injection of FITC-labeled dextran (Sigma-Aldrich) and immunohistochemical analysis after routine paraffin embedding.

Immunobistochemical analyses of CEACAM1 and PECAM1 expression. Immunohistochemical staining of CEACAM1 and PECAM1 was performed on cryosections or paraffin-embedded specimens with polyclonal anti-CEACAM1 antiserum (2456, 1:500 dilution; prepared by $\mathrm{N}$. Beauchemin) and with an anti-PECAM1 antibody $(2 \mu \mathrm{g} / \mathrm{ml}$, DPC Biermann). CEACAM1 and PECAM1 binding was visualized through alkaline phosphatase activity with either naphthol-AS-bisphosphate or Vector Blue (Vector Laboratories) on paraffin sections and FITC-labeled anti- 
rabbit or Cy3-labeled anti-rat antibodies (Vector Laboratories) on cryosections. Counterstaining of nuclei was performed with either Mayer's hemalaun or Nuclear Fast Red (Sigma-Aldrich).

Electron microscopy. Matrigel plugs were fixed in $4^{\circ} \mathrm{C}$ phosphate-buffered glutaraldehyde (3.5\%, $\mathrm{pH} 7.4$ ) for 12 hours and processed for electron microscopy as described previously (50). Contrasted sections were viewed in a Philips CM100 electron microscope.

Induction of hind limb ischemia and evaluation of collateral growth. All experiments were performed according to section 8 of the German Law for the Protection of Animals and were approved by the Behörde für Wissenschaft und Gesundheit, Lebensmittelsicherheit und Veterinärwesen, Hamburg, Germany, and the Review Board at the Animal Center, University Medical Center Hamburg-Eppendorf. CEACAM1 ${ }^{\text {endo+ }}$ and Ceacam 1-/- mice and their appropriate WT littermates were anesthetized with isoflurane inhalation. The femoral artery was ligated unilaterally between the origin of the deep femoral artery and the entry of the lateral circumflex artery, which was also ligated. A second ligation was placed about $1 \mathrm{~cm}$ below the first ligation using 7-0 silk sutures (Ethicon). After 7 days of femoral artery occlusion, mice were anesthetized again. The external iliac artery was prepared, and a transonic flow probe was placed around the vessel for the recording of blood flow amplitudes. Subsequently a left-sided thoracotomy was performed, and ultrasonicated red fluorescent microspheres ( $15 \mu \mathrm{m}$; Invitrogen Corp.) were injected into the beating left ventricle as described previously (32). Microspheres were counted under the fluorescent microscope in $70-\mu \mathrm{m}$ sections from the gastrocnemial muscle. At fixed intervals, $10-\mu \mathrm{m}$ sections were obtained for immunohistochemical analyses. Kidneys served as reference organs proximal to the occlusion site. All experiments and data analysis were performed blinded, without knowledge of the type of mice investigated. Collateral blood flow was expressed as occluded and nonoccluded hind limb perfusion ratios (microspheres) and maximum blood flow amplitude (transonic flow probe) to the ischemic leg (32).
Statistics. Statistical analyses were carried out with 2-tailed Student's $t$ test. $P$ values less than 0.05 were considered to be statistically significant.

\section{Acknowledgments}

This work was supported by the Deutsche Forschungsgemeinschaft (grant SFB470-C5) and the Deutsche Krebshilfe (grant 101723-Wa4) to C. Wagener, the Deutsche Forschungsgemeinschaft (grants It 13/1-3 and It 13/2-3 within the priority program "Angiogenesis” SPP 1069) to W.D. Ito, the Cancer Research Society Inc. and the Canadian Institutes for Health Research (grant 42501) to N. Beauchemin, and the Heinz-Breuer-Stipendium of the German Society for Clinical Chemistry and Laboratory Medicine and the Canadian Cancer Research Society postdoctoral fellowship to A.K. Horst. The authors also gratefully acknowledge expert technical assistance by Roswitha Reusch, Elke Schäfer, Susanne Feldhaus, Christa Frenz, Petra Tide, and Krimhild Scheike. The authors also wish to thank Thomas N. Sato for the kind gift of the pHHNS plasmid.

Received for publication December 27, 2004, and accepted in revised form March 7, 2006.

Address correspondence to: C. Wagener, Department of Clinical Chemistry, Center of Clinical Pathology, University Medical Center Hamburg-Eppendorf, Martinistrasse 52, D-20246 Hamburg, Germany. Phone: 49-40-42803-2981; Fax: 49-40-42803-4621; E-mail: wagener@uke.uni-hamburg.de.

Andrea Kristina Horst and Wulf D. Ito contributed equally to this work.

Nicole Beauchemin and Cristophe Wagener contributed equally to this work.
1. Hanahan, D., and Folkman, J. 1996. Patterns and emerging mechanisms of the angiogenic switch during tumorigenesis. Cell. 86:353-364.

2. Folkman, J. 2002. Role of angiogenesis in tumor growth and metastasis. Semin. Oncol. 29:15-18.

3. Carmeliet, P. 2005. Angiogenesis in life, disease and medicine. Nature. 438:932-936.

4. Carmeliet, P. 2000. Mechanisms of angiogenesis and arteriogenesis. Nat. Med. 6:389-395.

5. Ferrara, N. 2004. Vascular endothelial growth factor: basic science and clinical progress. Endocr. Rev 25:581-611.

6. Jain, R.K. 2005. Normalization of tumor vasculature: an emerging concept in antiangiogenic therapy. Science. 307:58-62.

7. Ferrara, N., and Kerbel, R.S. 2005. Angiogenesis as a therapeutic target. Nature. 438:967-974.

8. DeLisser, H.M., et al. 1997. Involvement of endothelial PECAM-1/CD31 in angiogenesis. Am. J. Pathol. 151:671-677.

9. Zhou, Z., Christofidou-Solomidou, M., Garlanda, C., and DeLisser, H.M. 1999. Antibody against murine PECAM-1 inhibits tumor angiogenesis in mice. Angiogenesis. 3:181-188

10. Ishida, T., et al. 2003. Targeted disruption of endothelial cell-selective adhesion molecule inhibits angiogenic processes in vitro and in vivo. J. Biol. Chem. 278:34598-34604.

11. Jones, N., Iljin, K., Dumont, D.J., and Alitalo, K. 2001. Tie receptors: new modulators of angiogenic and lymphangiogenic responses. Nat. Rev. Mol. Cell Biol. 2:257-267.

12. Prall, F., et al. 1996. CD66a (BGP), an adhesion molecule of the carcinoembryonic antigen family, is expressed in epithelium, endothelium, and myeloid cells in a wide range of normal human tissues. J. Histochem. Cytochem. 44:35-41.

13. Ergun, S., et al. 2000. CEA-related cell adhesion molecule 1: a potent angiogenic factor and a major effector of vascular endothelial growth factor. $\mathrm{Mol}$. Cell. 5:311-320.

14. Sawa, H., et al. 1997. Spatiotemporal expression of C-CAM in the rat placenta. J. Histochem. Cytochem. 45:1021-1034.

15. Chalajour, F., et al. 2004. Angiogenic activation of valvular endothelial cells in aortic valve stenosis. Exp. Cell Res. 298:455-464.

16. Kilic, N., et al. 2005. Pro-angiogenic signaling by the endothelial presence of CEACAM1. J. Biol. Chem. 280:2361-2369.

17. Oh, P., et al. 2004. Subtractive proteomic mapping of the endothelial surface in lung and solid tumours for tissue-specific therapy. Nature. 429:629-635

18. Hong, Y.K., et al. 2004. Lymphatic reprogramming of blood vascular endothelium by Kaposi sarcomaassociated herpesvirus. Nat. Genet. 36:683-685.

19. Chen, W.J., et al. 2005. Gene expression profiles in hypoxic preconditioning using cDNA microarray analysis: altered expression of an angiogenic factor, carcinoembryonic antigen-related cell adhesion molecule 1. Shock. 24:124-131.

20. Wagener, C., and Ergun, S. 2000. Angiogenic properties of the carcinoembryonic antigen-related cell adhesion molecule 1. Exp. Cell Res. 261:19-24.

21. Gehling, U.M., et al. 2000. In vitro differentiation of endothelial cells from AC133-positive progenitor cells. Blood. 95:3106-3112.

22. Horst, A.K., and Wagener, C. 2004. CEA-related CAMs. In Handbook of experimental pharmacology.
J. Behrens and W.J. Nelson, editors. Springer. New York, New York, USA. 283-341.

23. Bamberger, A.M., et al. 2000. The adhesion molecule CEACAM1 (CD66a, C-CAM, BGP) is specifically expressed by the extravillous intermediate trophoblast. Am. J. Pathol. 156:1165-1170.

24. Brummer, J., et al. 2001. cis Interaction of the cell adhesion molecule CEACAM1 with integrin beta(3). Am. J. Pathol. 159:537-546.

25. Ebrahimnejad, A., et al. 2004. CEACAM1 enhances invasion and migration of melanocytic and melanoma cells. Am. J. Pathol. 165:1781-1787.

26. Najjar, S.M. 2002. Regulation of insulin action by CEACAM1. Trends Endocrinol. Metab. 13:240-245.

27. Abou-Rjaily, G.A., et al. 2004. CEACAM1 modulates epidermal growth factor receptor-mediated cell proliferation. J. Clin. Invest. 114:944-952. doi:10.1172/JCI200421786.

28. Muller, M.M., Singer, B.B., Klaile, E., Obrink, B., and Lucka, L. 2005. Transmembrane CEACAM1 affects integrin-dependent signaling and regulates extracellular matrix protein-specific morphology and migration of endothelial cells. Blood. 105:3925-3934.

29. Leung, N., et al. 2006. Deletion of the carcinoembryonic antigen-related cell adhesion molecule 1 (Ceacam1) gene contributes to colon cancer progression in a murine model of carcinogenesis. Oncogene. In press.

30. Schlaeger, T.M., et al. 1997. Uniform vascularendothelial-cell-specific gene expression in both embryonic and adult transgenic mice. Proc. Natl. Acad. Sci. U. S. A. 94:3058-3063.

31. Nicosia, R.F., and Ottinetti, A. 1990. Growth of microvessels in serum-free matrix culture of rat 
aorta. A quantitative assay of angiogenesis in vitro. Lab. Invest. 63:115-122.

32. Jacobi, J., et al. 2004. Adenoviral gene transfer with soluble vascular endothelial growth factor receptors impairs angiogenesis and perfusion in a murine model of hindlimb ischemia. Circulation. 110:2424-2429.

33. Ito, W.D., et al. 1997. Monocyte chemotactic protein-1 increases collateral and peripheral conductance after femoral artery occlusion. Circ. Res. 80:829-837.

34. Scholz, D., et al. 2002. Contribution of arteriogenesis and angiogenesis to postocclusive hindlimb perfusion in mice. J. Mol. Cell. Cardiol. 34:775-787.

35. Robitaille, J., et al. 1999. Comparison of expression patterns and cell adhesion properties of the mouse biliary glycoproteins Bbgp1 and Bbgp2. Eur. J. Biochem. 264:534-544.

36. Cao, G., et al. 2002. Involvement of human PECAM-1 in angiogenesis and in vitro endothelial cell migration. Am. J. Physiol. Cell Physiol. 282:C1181-C1190.

37. Carmeliet, P., et al. 1999. Targeted deficiency or cytosolic truncation of the VE-cadherin gene in mice impairs VEGF-mediated endothelial survival and angiogenesis. Cell. 98:147-157.

38. Yang, J.T., Rayburn, H., and Hynes, R.O. 1993. Embryonic mesodermal defects in alpha 5 integrin-deficient mice. Development. 119:1093-1105.

39. Senger, D.R., et al. 1983. Tumor cells secrete a vascular permeability factor that promotes accumulation of ascites fluid. Science. 219:983-985.

40. Bello, L., et al. 2001. Alpha(v)beta3 and alpha(v)beta5 integrin expression in glioma periphery. Neurosurgery. 49:380-389; discussion 390.

41. Brooks, P.C., Clark, R.A., and Cheresh, D.A. 1994. Requirement of vascular integrin alpha v beta 3 for angiogenesis. Science. 264:569-571.

42. Petitclerc, E., et al. 1999. Integrin alpha(v)beta3 promotes M21 melanoma growth in human skin by regulating tumor cell survival. Cancer Res. 59:2724-2730.

43. Rehn, M., et al. 2001. Interaction of endostatin with integrins implicated in angiogenesis. Proc. Natl. Acad. Sci. U. S. A. 98:1024-1029.

44. Kunath, T., Ordonez-Garcia, C., Turbide, C., and Beauchemin, N. 1995. Inhibition of colonic tumor cell growth by biliary glycoprotein. Oncogene.
11:2375-2382.

45. Huber, M., et al. 1999. The carboxyl-terminal region of biliary glycoprotein controls its tyrosine phosphorylation and association with protein-tyrosine phosphatases SHP-1 and SHP-2 in epithelial cells. J. Biol. Chem. 274:335-344.

46. McCuaig, K., Rosenberg, M., Nedellec, P., Turbide, C., and Beauchemin, N. 1993. Expression of the Bgp gene and characterization of mouse colon biliary glycoprotein isoforms. Gene. 127:173-183.

47. Teichert-Kuliszewska, K., et al. 2001. Biological action of angiopoietin-2 in a fibrin matrix model of angiogenesis is associated with activation of Tie2. Cardiovasc. Res. 49:659-670.

48. Kuhlencordt, P.J., et al. 2004. Role of endothelial nitric oxide synthase in endothelial activation: insights from eNOS knockout endothelial cells. Am. J. Physiol. Cell Physiol. 286:C1195-C1202.

49. Passaniti, A. 1992. Extracellular matrix-cell interactions: Matrigel and complex cellular pattern formation. Lab. Invest. 67:804; author reply 804-808.

50. Luft, J.H. 1961. Improvements in epoxy resin embedding methods. J. Biophys. Biochem. Cytol. 9:409-414. 